\title{
A Machine Learning Approach to Passively Informed Prediction of Mental Health Risk in People with Diabetes: Retrospective Case-Control Analysis
}

Jessica Yu, PhD; Carter Chiu, PhD; Yajuan Wang, PhD; Eldin Dzubur, PhD; Wei Lu, PhD; Julia Hoffman, PsyD Livongo Health, Inc, Mountain View, CA, United States

\section{Corresponding Author:}

Jessica $\mathrm{Yu}, \mathrm{PhD}$

Livongo Health, Inc

150 W Evelyn Ave

Ste 150

Mountain View, CA, 94041

United States

Phone: 16508048434

Email: jessica.yu@livongo.com

\section{Abstract}

Background: Proactive detection of mental health needs among people with diabetes mellitus could facilitate early intervention, improve overall health and quality of life, and reduce individual and societal health and economic burdens. Passive sensing and ecological momentary assessment are relatively newer methods that may be leveraged for such proactive detection.

Objective: The primary aim of this study was to conceptualize, develop, and evaluate a novel machine learning approach for predicting mental health risk in people with diabetes mellitus.

Methods: A retrospective study was designed to develop and evaluate a machine learning model, utilizing data collected from 142,432 individuals with diabetes enrolled in the Livongo for Diabetes program. First, participants' mental health statuses were verified using prescription and medical and pharmacy claims data. Next, four categories of passive sensing signals were extracted from the participants' behavior in the program, including demographics and glucometer, coaching, and event data. Data sets were then assembled to create participant-period instances, and descriptive analyses were conducted to understand the correlation between mental health status and passive sensing signals. Passive sensing signals were then entered into the model to train and test its performance. The model was evaluated based on seven measures: sensitivity, specificity, precision, area under the curve, $\mathrm{F}_{1}$ score, accuracy, and confusion matrix. SHapley Additive exPlanations (SHAP) values were computed to determine the importance of individual signals.

Results: In the training (and validation) and three subsequent test sets, the model achieved a confidence score greater than 0.5 for sensitivity, specificity, area under the curve, and accuracy. Signals identified as important by SHAP values included demographics such as race and gender, participant's emotional state during blood glucose checks, time of day of blood glucose checks, blood glucose values, and interaction with the Livongo mobile app and web platform.

Conclusions: Results of this study demonstrate the utility of a passively informed mental health risk algorithm and invite further exploration to identify additional signals and determine when and where such algorithms should be deployed.

(J Med Internet Res 2021;23(8):e27709) doi: 10.2196/27709

\section{KEYWORDS}

diabetes mellitus; mental health; risk detection; passive sensing; ecological momentary assessment; machine learning

\section{Introduction}

In the United States, 34.2 million people are affected by diabetes mellitus [1]. Approximately $25 \%$ of those living with diabetes experience significant depressive symptoms, and up to $40 \%$ experience generalized anxiety disorder (GAD) [1-3]. Individuals with diabetes and mental health challenges have been found to be less adherent to diabetes treatment recommendations, including diet, exercise, medication use, glucose monitoring, and medical appointments, and they are at a greater risk for adverse medical outcomes [1]. Health 
care costs for those with comorbid diabetes and mental health have been estimated to be US \$4 billion to \$9 billion greater than for those without these conditions [4]. However, proactive detection of mental health needs of people with diabetes could facilitate early intervention, thereby improving their overall health and quality of life and reducing the health and economic burdens placed on this population and the health care system as a whole.

Despite recommendations by the American Diabetes Association and the United States Preventive Services Task Force to routinely evaluate people with diabetes for their mental health needs, only $25 \%$ to $50 \%$ of people with diabetes who have depression receive a mental health diagnosis and intervention $[5,6]$. This gap in receiving care is a result of a shortage of mental health professionals available to offer assessment and intervention, a lack of mental health knowledge among primary care providers who most often care for patients with diabetes, and limited access to mental health screening tools in health care practices offering services to these patients [6]. Newer methods such as passive sensing and ecological momentary assessments (EMAs) provide a more scalable, less effort- and time-intensive approach to information gathering and assessment. Passive sensing refers to the capture of data about a person without any extra effort on their part [7]. EMA refers to the repeated sampling of an individual's behavior in real time within their natural environment [8]. Both methods can be integrated into or with devices and services that people with diabetes already utilize in their daily lives, such as blood glucose meters, smartphones, and health coaching platforms to enable the collection and processing of data in real time and to provide context for real-time interventions [7].

Although passive sensing and EMA have previously been examined in the general population, limited studies have focused on the detection of mental health needs among the diabetes population outside of using smartphones as data warehouses, relying on accelerometer, GPS, ambient light sensors, and call $\log$ data $[9,10]$. Moreover, no known study to date has attempted to detect mental health concerns in people with diabetes by using blood glucose meters despite the fact that individuals living with diabetes are encouraged to engage with these devices at regular intervals, blood glucose monitoring has been found to be correlated with psychological effects, and engagement with these devices and testing blood glucose levels are known to be associated with mood or stress [11-13]. Further, blood glucose meter data can be paired with data from other sources for a robust view of a person's behavioral and emotional profile. The primary aim of this study was to conceptualize, develop, and evaluate a novel approach using passive sensing for predicting mental health risk in people with diabetes.

\section{Methods}

\section{Study Design}

A multidisciplinary team of experts in data science, machine learning, and clinical and experimental psychology collaborated in the development of a machine learning model for detecting potential mental health risk from passive sensing signals that was both clinically relevant and statistically rigorous. A retrospective analysis was performed to evaluate the machine learning model for detecting potential mental health risk from passive sensing signals leveraging data collected during participants' engagement in the Livongo for Diabetes program [14].

\section{Livongo for Diabetes}

The Livongo for Diabetes program is a digital remote program for the management of chronic condition focused on empowering members by providing education and tools to self-manage their diabetes through mobile technology. The program offers members (1) a cellular-enabled, two-way messaging device that measures blood glucose and delivers personalized insights; (2) free, unlimited blood glucose test strips; (3) real-time support from diabetes response specialists available 24 hours a day, 7 days a week, 365 days a year; and (4) access to certified diabetes care and education specialists for support and goal setting. Further details on the Livongo for Diabetes program and its efficacy in improving diabetes-related outcomes are available in the literature [15-17].

\section{Study Participants}

Study participants were defined as those individuals enrolled in the Livongo for Diabetes Program between January 1, 2018, and February 28, 2020, who used their blood glucose meter at least once $(\mathrm{N}=142,432)$. Approval was granted by the Aspire Independent Review Board (\#520160099). All participants provided consent to participate, and guidelines outlined in the Declaration of Helsinki were followed.

\section{Study Procedure}

The mental health status of each participant was verified through available data from two sources that included data on medications prescribed to and filled by participants and mental health-related interventions. Next, passive sensing signals were extracted from participants' behaviors as they interacted with Livongo's blood glucose meter, mobile app, web portal, and coaching feature. Then, data sets were assembled by aggregating these signals per participant over various periods, creating participant-period instances. Descriptive analyses were conducted to understand the correlation between the signals and mental health status. Finally, demographic information and passive sensing signals were entered into the model, training it to understand the relationships between these signals and the participants' mental health status.

\section{Study Population Identification}

Identification of population cases and controls with respect to mental health conditions - which, in the context of labeling data for model training, we refer to as ground truth-was performed utilizing two data sources: (1) claims data and (2) medication prescription data. Claims data contained information on medications indicated for mental health conditions that were prescribed to and filled by participants, as well as mental health-related assessments and interventions. Based on data availability and right to use, $6.1 \%(8633 / 142,432)$ of the study participants had claims data, which provided a diverse way to identify their mental health needs through diagnoses, procedures, and prescriptions. Medication prescription data contained only information on mental health-related medications that were 
prescribed to and filled by participants. Medication prescription data were available for the entire study population and used to identify cases for the remaining participants. Participant demographic characteristics, which were used to evaluate signals correlated with mental health in the study population, are summarized in Table 1.

Table 1. Participant demographics and characteristics at the time of enrollment $(\mathrm{N}=142,432)$.

\begin{tabular}{|c|c|}
\hline Characteristic & Value \\
\hline Age in years, mean (SD) & $54.8(12.4)$ \\
\hline \multicolumn{2}{|l|}{ Gender, n (\%) } \\
\hline Female & $68,968(48.4)$ \\
\hline Male & $73,147(51.6)$ \\
\hline Other & $317(0.22)$ \\
\hline \multicolumn{2}{|l|}{ Ethnicity, n (\%) } \\
\hline Hispanic & $12,809(9.0)$ \\
\hline Non-Hispanic & $86,116(60.5)$ \\
\hline Unknown & $43,507(30.6)$ \\
\hline \multicolumn{2}{|l|}{ Race, $n(\%)$} \\
\hline Caucasian & $66,551(46.7)$ \\
\hline Black or African American & $14,702(10.3)$ \\
\hline Asian & $8199(5.76)$ \\
\hline Pacific Islander & $468(0.33)$ \\
\hline American Indian & $725(0.51)$ \\
\hline Other & $6,588(4.63)$ \\
\hline Unknown & $45,199(31.7)$ \\
\hline \multicolumn{2}{|l|}{ Diabetes type, n (\%) } \\
\hline Type 1 & $14,360(10.1)$ \\
\hline Type 2 & $126,369(88.7)$ \\
\hline Unknown & $1603(1.2)$ \\
\hline Years since diagnosis, mean (SD) & $8.27(8.1)$ \\
\hline First reported $A_{1 c}$, mean $(S D)$ & $7.51(1.7)$ \\
\hline \multicolumn{2}{|l|}{ Insulin use, n (\%) } \\
\hline Yes & $39,153(27.5)$ \\
\hline No & $102,622(72.1)$ \\
\hline Unknown & $657(0.5)$ \\
\hline
\end{tabular}

\section{Passive Sensing Signals}

All data utilized in the study were collected in the course of how participants naturally engaged with the Livongo for Diabetes Program. That is, no data were collected solely for study purposes. We identified various data sources potentially useful to detect mental health risk behaviors. From these data sources, we extracted 83 individual signals that can be broadly classified into the following four categories. Note that individual signal names are withheld to protect proprietary information.

\section{Demographics}

Demographic factors such as age, gender, ethnicity, and race have been shown to be related to mental health [18]. Therefore, we included participants' demographic data into the model.

\section{Glucometer Data}

The Livongo blood glucose meter is the most frequent interaction point for participants of the Livongo for Diabetes Program. Low rates of blood glucose monitoring [2] and poorer blood glucose control [19] have been linked to depression among those with diabetes; and depression, anxiety, and stress symptoms are greater among people with diabetes than those without [20]. Differences in device usage is particularly informative of conditions such as depression when examining 
usage time of day [10] and weekday [21]. Therefore, the key metrics derived from glucometer usage included the number of times blood glucose was checked; blood glucose levels; and variations, responses to questions to assess context such as current emotional state, and time of the day and day of week when the reading was taken.

\section{Coaching Data}

In the Livongo for Diabetes Program, Livongo coaches contact individuals under certain conditions. Numerous studies have affirmed relationships between sociability and mental health. Fewer calls and fewer incoming texts have been linked to depression [22], whereas frequency and duration of conversations have been shown to be useful in evaluation of bipolar disorder [23]. Coaching data can serve as a proxy for sociability, for which successful or failed contacts and time spent interacting can be used to glean valuable insights.

\section{Event Data}

In addition to the blood glucose meter, individuals enrolled in the Livongo for Diabetes Program interact with multiple platforms during program participation, including the Livongo mobile app and web portal. In following the motivations behind utilizing glucometer and coaching data, we collected frequency, duration, interactivity, and consistency of interaction sessions, as well as the time of day and day of week information associated with the use of the mobile app and web portal. In addition, we tracked voluntary report sharing with friends and family as well as interactions with pop-up reminders.

\section{Statistical Analyses}

We conducted correlation analyses using Pearson $r$ to preliminarily gauge the strength of the relationship between each extracted signal and the presence of mental health conditions. Among the most highly correlated signals, demographics were well represented, including gender (male: $r=-0.156, P<.001$; female: $r=0.155, P<.001$ ), race (Asian: $r=-0.104, P<.001$; White: $r=0.101, P<.001$; Black: $r=-0.041$, $P<001)$, BMI $(r=0.086, P<.001)$, and smoking status (active smoker: $r=0.047, P<.001$ ). With regard to glucometer, coaching, and event data, responses on current emotional state from participants during blood glucose checks indicating wellness ( $r=-0.108, P<.001)$ or unwellness $(r=0.121, P<.001)$ were most strongly correlated. Greater frequency and consistency of interactions with services were negatively associated with mental health conditions. In addition, we found that greater variation in blood glucose values, as measured by $\mathrm{SD}$, were positively correlated with mental health conditions $(r=0.041, P<.001)$.

\section{Outcome Data}

When quantifying the performance of a prospective model for identifying mental health risk, it is important to consider a variety of perspectives. Metrics such as total accuracy are inadequate if used alone because they can hide model deficiencies on imbalanced data. Consider these three questions, which cannot be answered with total accuracy alone but are of particular importance in a diagnostic setting:

1. How often are those with mental health needs (cases) correctly identified?
2. How often are those without mental health needs (controls) correctly identified?

3. How often are those with predicted mental health needs truly cases?

The following seven measures commonly used in machine learning model evaluation [24] were selected to address the above questions and beyond, enabling a holistic view of model performance:

1. Sensitivity or recall, which addresses question 1

2. Specificity, which addresses question 2

3. Precision, which addresses question 3

4. Area under the curve (AUC), defined as the area under the receiver operating characteristic curve-an important measure quantifying the model's capacity to differentiate cases and controls (1=ideal performance, $0.5=$ random prediction)

5. $\mathrm{F}_{1}$ score, defined as the harmonic mean of the precision and recall

6. Accuracy, defined as the proportion of instances correctly classified

7. The confusion matrix, which depicts the number of correctly and incorrectly identified cases and controls

\section{Model Development}

To develop our machine learning model, we then had to divide the study population into two segments. The first segment, termed the training and validation set, enabled the model to learn. The second segment, termed the test sets, was held separate from model training and used to evaluate the model's ability to generalize to unseen data. For training, we used a time-interval slice of the population consisting of 124,322 participants (ie, $87 \%$ of the study population) who had activated their blood glucose meters in 2018 or 2019, with passive sensing signal data collected in that timeframe. For testing, we defined three distinct test sets designed to comprehensively evaluate model performance. The first two test sets used medication prescription or refill data as their sources of ground truth, whereas the third test set used medical and pharmacy claims data as its source of ground truth.

1. The first test set (test set 1) consisted of the same participant subset as the training data, but with signal data collected in the first two months of 2020. This data subset evaluated model prediction capability on previously seen participants $(93,155 / 142,432,65.4 \%)$.

2. The second test set (test set 2) consisted of participants activated in the first two months of 2020 and the associated signal data. This test set evaluated model prediction capability on new, unseen participants $(9477 / 142,432$, $6.7 \%)$.

3. The third test set (test set 3) utilized the claims data by identifying participants activated in 2018,2019 , or the first two months of 2020. This final data subset evaluated prediction capability with regard to unseen participants, with mental health needs identified through more diverse sources beyond prescriptions only $(8633 / 142,432,6.1 \%)$.

A visual summary of the data subsets is presented in Figure 1, and the specific numeric breakdowns are described in Table 2. 
Furthermore, the demographic information for each subset is detailed in Multimedia Appendix 1.

To increase the model utility, passive signals were aggregated during a certain period (participant-period) and presented to the model for prediction of mental health risk. We defined the participant-period as an instance. In this study, an aggregation window of 4 weeks was selected to optimize data availability. Furthermore, two additional conditions were applied to filter out ineligible participant-period instances: (1) instances before a participant had participated in the Livongo Program and (2) instances of extended inactivity, defined as 30 or more days without any interaction with the Livongo for Diabetes Program.
The rationale for the first criterion is trivial. The second eligibility condition reflects the reasoning that a model for identifying mental health needs from passive signals should only be employed when a signal is present, specifically signals where missingness cannot be assumed to be zero (eg, blood glucose values). Thus, our model should only be trained and evaluated on complete instances. Table 3 demonstrates the distribution of eligible instances among the data subsets. Note the class imbalance, with control instances represented at roughly a 2:1 ratio over case instances in each data subset.

Figure 1. Source and date ranges of data subsets defined in this study.

\section{Prescription data}

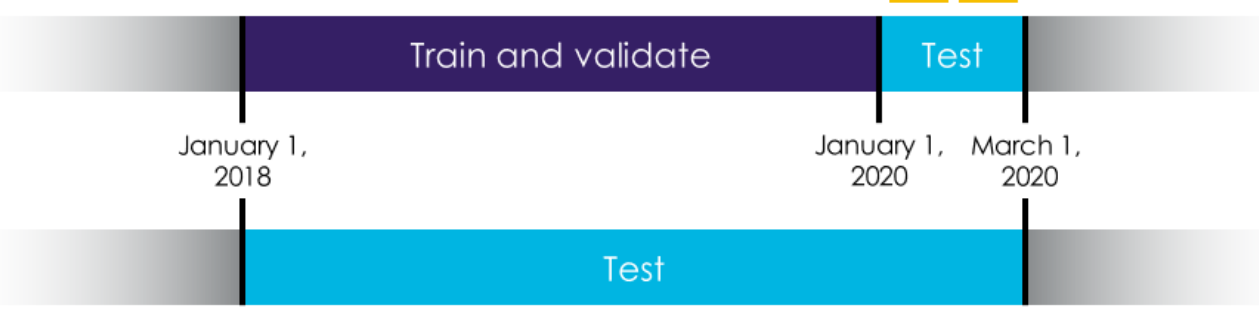

Claims data

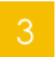

Table 2. Number of participant cases and controls for each data subset.

\begin{tabular}{llll}
\hline Data subset & Cases, $\mathrm{n}(\%)$ & Controls, $\mathrm{n}(\%)$ & Subset total, $\mathrm{n}$ \\
\hline Training or validation set & $42,481(34.2)$ & $81,841(65.8)$ & 124,322 \\
Test set 1 & $31,251(33.5)$ & $61,904(66.5)$ & 93,155 \\
Test set 2 & $2919(30.8)$ & $6558(69.2)$ & 9477 \\
Test set 3 & $3358(38.9)$ & $5275(61.1)$ & 8633 \\
Total unique & $48,758(34.2)$ & $93,674(65.8)$ & 142,432 \\
\hline
\end{tabular}

Table 3. Number of participant-period cases and controls for each data subset.

\begin{tabular}{llll}
\hline Data subset & Cases, $\mathrm{n}(\%)$ & Controls, $\mathrm{n}(\%)$ & Subset total, $\mathrm{n}$ \\
\hline Training and validation set & $287,311(34.3)$ & $549,183(65.7)$ & 836,494 \\
Test set 1 & $54,709(33.3)$ & $109,550(66.7)$ & 164,259 \\
Test set 2 & $2953(30.8)$ & $6640(69.2)$ & 9593 \\
Test set 3 & $34,190(40.1)$ & $51,150(59.9)$ & 85,340 \\
Total & $379,163(34.6)$ & $716,523(65.4)$ & $1,095,686$
\end{tabular}

A machine learning model was enlisted to capture the relationship between input activity features and exhibited mental health needs. The core component of our approach was the training of LightGBM [25] gradient tree boosting models on random subsets of the training data. This approach addressed the class imbalance; we undersampled the training control instances by random undersampling, thus reducing the number of control instances to equal the number of case instances. Because this technique reduced the number of control instances by roughly half, we saw the opportunity to train multiple models on multiple random subsets. This strategy enabled us to fully utilize the entire training data set, with each model training on a differing perspective of the data. We utilized soft voting to obtain an output prediction for a given instance, meaning the outputs of each constituent model-a value from 0 to 1 interpretable as the confidence that an instance is a case-were averaged to obtain a single aggregate confidence score.

Our final devised model consists of an ensemble of 10 LightGBM models. During our model selection process, we evaluated multiple other classes of machine learning models, including logistic regression, random forests, and neural networks. We also experimented with other flavors of gradient boosting, including XGBoost and CatBoost, and overall, 
LightGBM yielded the highest performance for our training task. To tune each constituent model, we used automated hyperparameter tuning enabled by the hyperopt [26] Python library with 5-fold cross-validation on the training set. Following this training procedure, we evaluated the model on the three held-out test sets to assess model performance.

\section{Results}

The results of our model's performance on each of the previously described data subsets are presented in Table 4. In addition, the associated confusion matrices are shown in Figure 2 , with both counts and percentages (normalized by class support size) depicted. Across all three test sets, the vast majority of metrics exceeded 0.5. Notably, we achieved an AUC of nearly 0.7 on the first test set and exceeded 0.65 across all three sets. The metrics for which the model fell short of the 0.5 mark were precision in the first and second test sets and the $F_{1}$ score for the second test set. However, it is important to note that owing to class imbalance, 0.5 would not be the theoretical precision or the $\mathrm{F}_{1}$ score yielded by random prediction. Rather, the precision obtained by random prediction would be the proportion of cases, with the $\mathrm{F}_{1}$ score affected commensurately. In our case, baseline precision would be approximately 0.3331 for the first test set and 0.3078 for the second, both of which were well outperformed by the reported precisions of 0.4702 and 0.4164 . Likewise, the baseline theoretical $F_{1}$ score of 0.3810 was greatly outperformed by the reported 0.4953 on the second test set. Concerning improvement over random prediction overall, our model produced an approximately 14-point gain in precision for the first test set and an approximately 10-point gain for the second and third sets, whereas recall improved by 14 and 12 points and AUC improved by 20 and 16 points, respectively. These results demonstrate a respectable, generalizable performance and are an encouraging advancement towards practical passive mental health risk assessment at scale.

Table 4. Performance metrics for each data subset.

\begin{tabular}{lllllll}
\hline Data subset & Sensitivity & Specificity & Precision & AUC & F Fcore $^{\mathrm{a}}$ & Accuracy \\
\hline Training and validation set & 0.688 & 0.667 & 0.519 & 0.745 & 0.592 & 0.674 \\
Test set 1 & 0.639 & 0.640 & 0.470 & 0.696 & 0.542 & 0.640 \\
Test set 2 & 0.621 & 0.605 & 0.412 & 0.658 & 0.495 & 0.610 \\
Test set 3 & 0.625 & 0.596 & 0.508 & 0.656 & 0.561 & 0.608 \\
\hline
\end{tabular}

${ }^{\mathrm{a}} \mathrm{AUC}$ : area under the curve. 
Figure 2. Confusion matrices for each data subset.

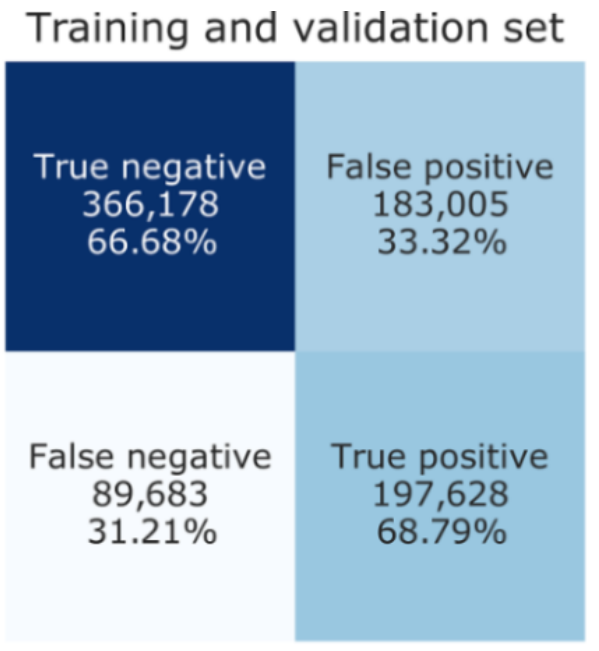

\section{Test set 2}

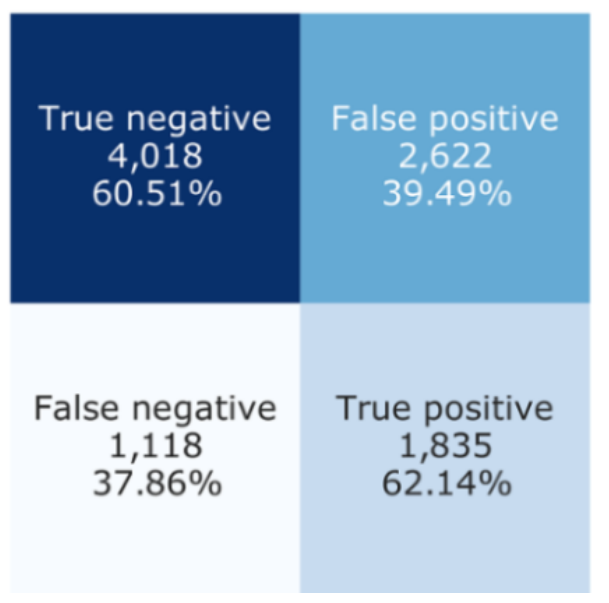

Our model results also provided the opportunity to gain further insight into the utility of the passive sensing signals. For this purpose, we used the SHapley Additive exPlanations (SHAP) method [27] to compute feature importance, allowing us to quantify the average contribution of each signal to the model. These values are presented in Table 5. Our findings closely mirrored the insights from our correlation analysis. Demographics ranked highly among signals, with gender and race identified as the two most relevant factors. Among passive

\section{Test set 1}

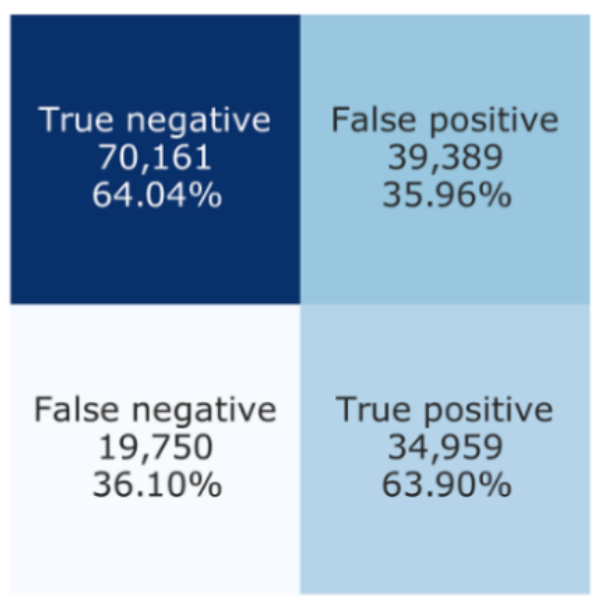

Test set 3

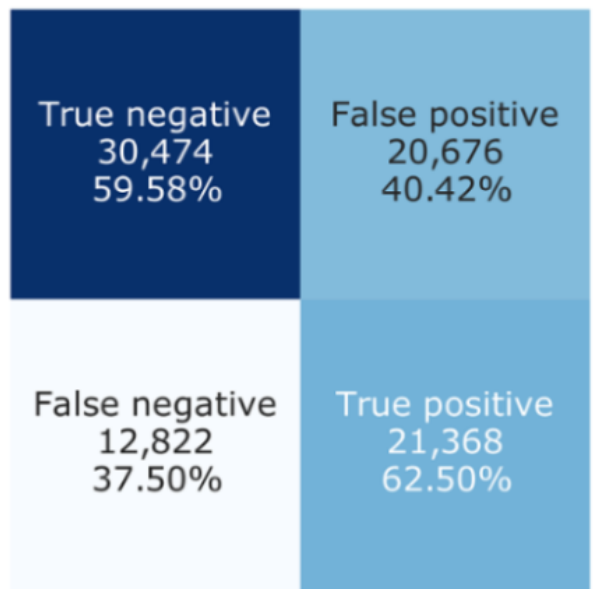

sensing signals, responses indicating an emotional state of wellness or unwellness during blood glucose checks were deemed the most important. Interaction frequency and consistency were also considered valuable according to SHAP. The model took into particular consideration blood glucose checks based on the times of day and the proportion of days they were performed. Mean and SD values of blood glucose levels also appeared in the top quartile of signals. 
Table 5. Feature importance as measured by mean absolute SHapley Additive exPlanations (SHAP) values, interpreted as the average impact on model output (log-odds) magnitude. Note that aliases based on the signal category are depicted in lieu of the signals to protect proprietary information.

\begin{tabular}{|c|c|}
\hline Passive signal & Mean absolute SHAP value \\
\hline Demographics 1 & 0.152 \\
\hline Demographics 2 & 0.089 \\
\hline Glucometer data 1 & 0.078 \\
\hline Demographics 3 & 0.040 \\
\hline Glucometer data 2 & 0.039 \\
\hline Demographics 4 & 0.037 \\
\hline Demographics 5 & 0.037 \\
\hline Event data 1 & 0.022 \\
\hline Demographics 6 & 0.018 \\
\hline Event data 2 & 0.017 \\
\hline Glucometer data 3 & 0.014 \\
\hline Glucometer data 4 & 0.013 \\
\hline Glucometer data 5 & 0.012 \\
\hline Glucometer data 6 & 0.012 \\
\hline Glucometer data 7 & 0.011 \\
\hline Demographics 7 & 0.011 \\
\hline Glucometer data 8 & 0.011 \\
\hline Glucometer data 9 & 0.010 \\
\hline Glucometer data 10 & 0.008 \\
\hline Glucometer data 11 & 0.008 \\
\hline Glucometer data 12 & 0.008 \\
\hline Glucometer data 13 & 0.007 \\
\hline Event data 3 & 0.006 \\
\hline Event data 4 & 0.006 \\
\hline Glucometer data 14 & 0.006 \\
\hline Glucometer data 15 & 0.006 \\
\hline Event data 5 & 0.006 \\
\hline Event data 6 & 0.005 \\
\hline Glucometer data 16 & 0.005 \\
\hline Event data 7 & 0.005 \\
\hline Event data 8 & 0.004 \\
\hline Event data 9 & 0.004 \\
\hline Event data 10 & 0.004 \\
\hline Glucometer data 17 & 0.004 \\
\hline Event data 11 & 0.003 \\
\hline Glucometer data 18 & 0.003 \\
\hline Coaching data 1 & 0.003 \\
\hline Glucometer data 19 & 0.002 \\
\hline Event data 12 & 0.002 \\
\hline Event data 13 & 0.002 \\
\hline Coaching data 2 & 0.002 \\
\hline
\end{tabular}




\begin{tabular}{|c|c|}
\hline Passive signal & Mean absolute SHAP value \\
\hline Event data 14 & 0.002 \\
\hline Event data 15 & 0.002 \\
\hline Glucometer data 20 & 0.002 \\
\hline Event data 16 & 0.002 \\
\hline Coaching data 3 & 0.001 \\
\hline Event data 17 & 0.001 \\
\hline Event data 18 & 0.001 \\
\hline Coaching data 4 & 0.001 \\
\hline Event data 19 & 0.001 \\
\hline Event data 20 & $<0.001$ \\
\hline Event data 21 & $<0.001$ \\
\hline Event data 22 & $<0.001$ \\
\hline Event data 23 & $<0.001$ \\
\hline Coaching data 5 & $<0.001$ \\
\hline Event data 24 & $<0.001$ \\
\hline Event data 25 & $<0.001$ \\
\hline Event data 26 & $<0.001$ \\
\hline Event data 27 & $<0.001$ \\
\hline Event data 28 & $<0.001$ \\
\hline Coaching data 6 & $<0.001$ \\
\hline Coaching data 7 & $<0.001$ \\
\hline Event data 29 & $<0.001$ \\
\hline Event data 30 & $<0.001$ \\
\hline Event data 31 & $<0.001$ \\
\hline Event data 32 & $<0.001$ \\
\hline Event data 33 & $<0.001$ \\
\hline Event data 34 & $<0.001$ \\
\hline Event data 35 & $<0.0001$ \\
\hline Event data 36 & $<0.0001$ \\
\hline Event data 37 & $<0.0001$ \\
\hline Event data 38 & $<0.0001$ \\
\hline Event data 39 & $<0.0001$ \\
\hline Event data 40 & $<0.0001$ \\
\hline Event data 41 & $<0.0001$ \\
\hline Event data 42 & $<0.0001$ \\
\hline Event data 43 & $<0.0001$ \\
\hline Event data 44 & $<0.0001$ \\
\hline Coaching data 8 & $<0.0001$ \\
\hline Coaching data 9 & 0 \\
\hline Coaching data 10 & 0 \\
\hline Coaching data 11 & 0 \\
\hline Event data 45 & 0 \\
\hline
\end{tabular}




\section{Discussion}

In this study, we found that a machine learning approach using passive sensing signals that included data on participant demographics, blood glucose meter use, interaction with diabetes coaches as a proxy for sociability, and engagement with the Livongo for Diabetes Program demonstrated utility in predicting mental health risk among people with diabetes.

The results of our approach invite further exploration and expansion. It is well understood that smartphones can be viewed as vehicles for passive data collection and help identify digital phenotypes of mental health disorders, as shown previously [28]. However, it is time to move beyond focusing on smartphones as the only devices that enable passive sensing and EMA and view other devices and services that people with diabetes must use for their self-management as robust data warehouses. In this particular study, participants who enrolled in the Livongo for Diabetes Program had access to a Bluetooth-enabled blood glucose meter for measuring their blood glucose levels, the Livongo mobile app and web platform for tracking food intake and physical activity as well as receiving health reminders, and Livongo coaches for coaching for diabetes self-management. Each device and service offered valuable data to input in our model. The blood glucose meters provided access to the participants' behavioral, emotional, and physiological data, such as how they were feeling at the time of measuring their blood glucose level and the reading itself. The Livongo mobile app and web platform enabled us to understand when participants were awake, using their smartphones, and engaged in a health-related activity. Coaching allowed us to understand whether participants were actively communicating with others. Together, these different data sources enabled us to create a data set that combined behavioral, emotional, and physiological factors into a holistic predictive algorithm. Although these particular devices and services are unique to Livongo members, there are ways to obtain similar data in the real world. For example, several commercially available wireless and Bluetooth-enabled blood glucose meters connect to mobile apps that enable people with diabetes mellitus to track and receive feedback on their blood glucose levels and share data with others. Such meters and associated apps host behavioral, communication, and physiological data similar to what we used in our model. There is also a plethora of health-related apps that enable individuals to track their food intake, physical activity, mood, sleep, and other health signals. These apps host additional behavioral and emotional data similar to what we used in our model.

Identifying potential mental health risk from passively collected signals is undoubtedly not a simple task, and our study has some limitations. First, because we limited our extracted signals to interactions with Livongo devices and applications, we did not have access to certain passive signals shown to be predictive in previous studies, such as mobile device accelerometer, ambient light sensor, or GPS data. As a result, our model was given a somewhat restricted view of a member's activity and sociability patterns. Second, we had access to a limited volume of medical and pharmacy claims data, which made it difficult to utilize the data on their own. However, both limitations could also be seen as strengths of our study. Our inability to access previously studied passive signals afforded us an opportunity to examine new signals from devices and services that are unique to people with diabetes. It may also be more acceptable from a privacy perspective. Furthermore, we had limited claims data; nevertheless, the data enabled us to confidently label participants as cases versus controls. Finally, a major strength of our study was the fact that, by design, no data were collected with active participant input for the express purpose of detecting mental health risk. In that regard, our proposed approach makes real-world deployment more readily feasible, in contrast to other studies of passive sensing and EMA for mental health, which required active participant participation and extensive sensor infrastructure.

In sum, our model is a bold step toward detecting potential mental health risk passively and autonomously. In its nascent stage, we recommend integrating such a model with existing systems and services, while continuing to improve the quality and completeness of care that can be offered to those dealing with mental health needs.

\section{Conflicts of Interest}

The authors own stock in Teladoc Health and are employees of Livongo Health, Inc.

\section{Multimedia Appendix 1}

Demographics distribution across various data sets.

[DOCX File, $20 \mathrm{~KB}-$ Multimedia Appendix 1]

\section{References}

1. National Diabetes Statistics Report, 2020. Centers for Disease Control and Prevention. URL: $\underline{\text { https://www.cdc.gov/diabetes/ }}$ data/statistics-report/index.html [accessed 2020-12-01]

2. Holt RIG, de Groot M, Golden SH. Diabetes and Depression. Curr Diab Rep 2014 Apr 18;14(6):1-17. [doi: 10.1007/s 11892-014-0491-3]

3. Grigsby AB, Anderson RJ, Freedland KE, Clouse RE, Lustman PJ. Prevalence of anxiety in adults with diabetes: a systematic review. J Psychosom Res 2002 Dec;53(6):1053-1060. [doi: 10.1016/s0022-3999(02)00417-8] [Medline: 12479986]

4. Egede LE, Walker RJ, Bishu K, Dismuke CE. Trends in costs of depression in adults with diabetes in the united states: Medical Expenditure Panel Survey, 2004-2011. J Gen Intern Med 2016 Jun;31(6):615-622. [doi: 10.1007/s11606-016-3650-1] [Medline: 26969312] 
5. Diabetes and mental health. Centers for Disease Control and Prevention. URL: https://www.cdc.gov/diabetes/managing/ mental-health.html [accessed 2020-12-01]

6. Owens-Gary MD, Zhang X, Jawanda S, Bullard KM, Allweiss P, Smith BD. The importance of addressing depression and diabetes distress in adults with type 2 diabetes. J Gen Intern Med 2019 Feb;34(2):320-324 [FREE Full text] [doi: 10.1007/s11606-018-4705-2] [Medline: 30350030]

7. Cornet VP, Holden RJ. Systematic review of smartphone-based passive sensing for health and wellbeing. J Biomed Inform 2018 Jan;77:120-132 [FREE Full text] [doi: 10.1016/j.jbi.2017.12.008] [Medline: 29248628]

8. Shiffman S, Stone AA, Hufford MR. Ecological momentary assessment. Annu Rev Clin Psychol 2008;4:1-32. [doi: 10.1146/annurev.clinpsy.3.022806.091415] [Medline: 18509902 ]

9. Marsch LA. Digital health data-driven approaches to understand human behavior. Neuropsychopharmacology 2021 Jan;46(1):191-196 [FREE Full text] [doi: 10.1038/s41386-020-0761-5] [Medline: 32653896]

10. Sarda A, Munuswamy S, Sarda S, Subramanian V. Using passive smartphone sensing for improved risk stratification of patients with depression and diabetes: cross-sectional observational study. JMIR Mhealth Uhealth 2019 Jan 29;7(1):e11041 [FREE Full text] [doi: 10.2196/11041] [Medline: $\underline{\text { 30694197] }}$

11. AADE7 Self-Care Behaviors. Association of Diabetes Care \& Education Specialists. URL: https://www.diabeteseducator.org/ living-with-diabetes/aade7-self-care-behaviors [accessed 2020-12-01]

12. Patton SR, Clements MA. Psychological reactions associated with continuous glucose monitoring in youth. J Diabetes Sci Technol 2016 May;10(3):656-661 [FREE Full text] [doi: 10.1177/1932296816638109] [Medline: 26969141]

13. Fisher L, Polonsky W, Parkin CG, Jelsovsky Z, Amstutz L, Wagner RS. The impact of blood glucose monitoring on depression and distress in insulin-naïve patients with type 2 diabetes. Curr Med Res Opin 2011 Nov;27 Suppl 3:39-46. [doi: 10.1185/03007995.2011.619176] [Medline: 21916532]

14. Livongo.com. URL: https://www.livongo.com/ [accessed 2021-06-24]

15. Downing J, Bollyky J, Schneider J. Use of a connected glucose meter and certified diabetes educator coaching to decrease the likelihood of abnormal blood glucose excursions: the Livongo for Diabetes Program. J Med Internet Res 2017 Jul 11;19(7):e234 [FREE Full text] [doi: 10.2196/jmir.6659] [Medline: 28698167]

16. Bollyky JB, Bravata D, Yang J, Williamson M, Schneider J. Remote lifestyle coaching plus a connected glucose meter with certified diabetes educator support improves glucose and weight loss for people with type 2 diabetes. J Diabetes Res 2018;2018:3961730 [FREE Full text] [doi: 10.1155/2018/3961730] [Medline: 29888288]

17. Whaley CM, Bollyky JB, Lu W, Painter S, Schneider J, Zhao Z, et al. Reduced medical spending associated with increased use of a remote diabetes management program and lower mean blood glucose values. J Med Econ 2019 Mar 08:1-9. [doi: 10.1080/13696998.2019.1609483] [Medline: 31012392]

18. Fleury M, Grenier G, Bamvita J, Perreault M, Jean-Caron. Typology of adults diagnosed with mental disorders based on socio-demographics and clinical and service use characteristics. BMC Psychiatry 2011 Apr 20;11(67):1-11. [doi:

10.1186/1471-244x-11-67]

19. Lustman PJ, Anderson RJ, Freedland KE, de Groot M, Carney RM, Clouse RE. Depression and poor glycemic control: a meta-analytic review of the literature. Diabetes Care 2000 Jul;23(7):934-942 [FREE Full text] [doi: 10.2337/diacare.23.7.934] [Medline: 10895843]

20. Krishna P. Depression, anxiety, and stress levels in patients with type 2 diabetes mellitus. Natl J Physiol Pharm Pharmacol 2018;8(9):1570-1572. [doi: 10.5455/njppp.2018.8.0929117092018]

21. Saeb S, Lattie EG, Schueller SM, Kording KP, Mohr DC. The relationship between mobile phone location sensor data and depressive symptom severity. PeerJ 2016;4:e2537 [FREE Full text] [doi: 10.7717/peerj.2537] [Medline: 28344895]

22. Ghandeharioun A, Fedor S, Sangermano L. Objective assessment of depressive symptoms with machine learning and wearable sensors data. 2017 Presented at: Seventh International Conference on Affective Computing and Intelligent Interaction; 2017; San Antonio, TX p. 325-332. [doi: 10.1109/acii.2017.8273620]

23. Abdullah S, Matthews M, Frank E, Doherty G, Gay G, Choudhury T. Automatic detection of social rhythms in bipolar disorder. J Am Med Inform Assoc 2016 May;23(3):538-543. [doi: 10.1093/jamia/ocv200] [Medline: 26977102]

24. Japkowicz N, Shah M. Evaluating learning algorithms: a classification perspective. New York, NY: Cambridge University Press; 2011.

25. Ke G, Meng Q, Finley T. LightGBM: a highly efficient gradient boosting decision tree. 2017 Presented at: 31 st Conference on Neural Information Processing Systems; 2017; Long Beach, CA.

26. Bergstra J, Yamins D, Cox D. Making a science of model search: hyperparameter optimization in hundreds of dimensions for vision architectures. 2013 Presented at: Proceedings of the 30th International Conference on Machine Learning; 2013; Atlanta, GA.

27. Lundberg S, Lee S. A unified approach to interpreting model predictions. 2017 Presented at: 31 st Conference on Neural Information Processing Systems; 2017; Long Beach, CA.

28. Zulueta J, Piscitello A, Rasic M, Easter R, Babu P, Langenecker SA, et al. Predicting mood disturbance severity with mobile phone keystroke metadata: a BiAffect digital phenotyping study. J Med Internet Res 2018 Jul 20;20(7):e241 [FRE Full text] [doi: 10.2196/jmir.9775] [Medline: 30030209] 


\section{Abbreviations}

AUC: area under the curve

EMA: ecological momentary assessment

GAD: generalized anxiety disorder

SHAP: SHapley Additive exPlanations

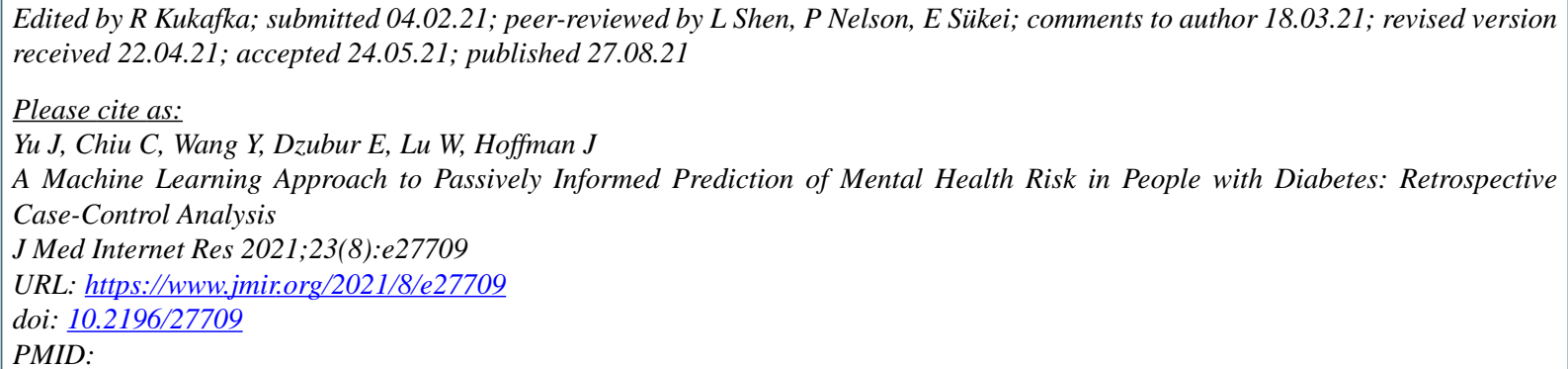

CJessica Yu, Carter Chiu, Yajuan Wang, Eldin Dzubur, Wei Lu, Julia Hoffman. Originally published in the Journal of Medical Internet Research (https://www.jmir.org), 27.08.2021. This is an open-access article distributed under the terms of the Creative Commons Attribution License (https://creativecommons.org/licenses/by/4.0/), which permits unrestricted use, distribution, and reproduction in any medium, provided the original work, first published in the Journal of Medical Internet Research, is properly cited. The complete bibliographic information, a link to the original publication on https://www.jmir.org/, as well as this copyright and license information must be included. 